\title{
Two LTR retrotransposon elements within the abscisic acid gene cluster in Botrytis cinerea B05.10, but not in SAS56
}

\author{
Ming Zhao \\ Chengdu Institute of Biology \\ Chinese Academy of Sciences \\ Chengdu 610041, PR China \\ Graduate University of Chinese Academy of Sciences \\ Beijing 100039, China \\ Tel: 8602885219314 \\ Fax: 8602885219314 \\ E-mail: zhaoming02292002@yahoo.com.cn
}

\section{Jin yan Zhou}

Chengdu Institute of Biology Chinese Academy of Sciences

Chengdu 610041, PR China

Tel: 8602885219314

Fax: 8602885219314

E-mail: abath@cib.ac.cn

\section{You jiu Tan}

Chengdu Institute of Biology

Chinese Academy of Sciences

Chengdu 610041, PR China

Graduate University of Chinese Academy of Sciences

Beijing 100039, China

Tel: 8602885219314

Fax: 8602885219314

E-mail: tanyou9@126.com

\section{Wei wei Song}

Chengdu Institute of Biology

Chinese Academy of Sciences

Chengdu 610041, PR China

Graduate University of Chinese Academy of Sciences

Beijing 100039, China

Tel: 8602885219314

Fax: 8602885219314

E-mail: songweiwei426@sohu.com

\section{Zhi dong Li}

Chengdu Institute of Biology

Chinese Academy of Sciences

Chengdu 610041, PR China

Tel: 8602885219314

Fax: 8602885219314

E-mail: abath@cib.ac.cn

\section{Hong Tan*}

Chengdu Institute of Biology Chinese Academy of Sciences

Chengdu 610041, PR China

Tel: 8602885219314

Fax: 8602885219314

E-mail: abath@cib.ac.cn

Financial support: Key Project of Chinese Academy of Sciences (No.KSCX2-YW-G016) and the Hi-Tech Research and Development Program (863) of China (No.2006AA10A213).

Keywords: Botrytis cinerea, genomic sequence analysis, in silico, LTR retrotransposon.

\footnotetext{
*Corresponding author
} 


\author{
Abbreviations: ABA: abscisic acid \\ IN: integrase domain \\ LTR: long terminal repeat \\ PBS: primer binding site \\ PPT: polypurine tract \\ $\mathrm{RT}$ : reverse transcriptase domain \\ TSD: target-site duplication \\ WGS: Whole Genome Shotgun
}

The plant hormone abscisic acid has huge economic potential and can be applied in agriculture and forestry for it is considered to be involved in plant resistance to stresses such as cold, heat, salinity, drought, pathogens and wounding. Now overproducing strains of Botrytis cinerea are used for biotechnological production of abscisic acid. An LTR retrotransposon, Boty-aba, and a solo LTR were identified by in silico genomic sequence analysis, and both were detected within the abscisic acid gene cluster in $B$. cinerea $B 05.10$, but not in $B$. cinerea SAS56. Boty-aba contains a pair of LTRs and two internal genes. The LTRs and the first gene have features characteristic of Ty3/gypsy LTR retrotransposons. The second gene is a novel gene, named brtn, which encodes for a protein (named BRTN) without putative conserved domains. The impressive divergence in structure of the abscisic acid gene clusters putatively gives new clues to investigate the divergence in the abscisic acid production yields of different $B$. cinerea strains.

The phytopathogenic ascomycete Botrytis cinerea, is known to produce the plant hormone abscisic acid (ABA), which plays a major role in several steps of plant growth and development, such as stomatal closure, embryo and seed dormancy, seed germination and the adaptation to environmental stress (Tudzynski and Sharon, 2002). ABA has huge economic potential and can be applied in agriculture and forestry for it is considered to be involved in plant resistance to stresses such as cold, heat, salinity, drought, pathogens and wounding (Tudzynski and Sharon, 2002). The production yields reported of $B$. cinerea ABA are $0 \sim 1.6 \mathrm{~g} / \mathrm{L}$ in axenic culture (Wu and Zheng, 1997; Tan and Li, 1998; Wu and Shi, 1998; Tudzynski and Sharon, 2002; Liang et al. 2004). Now overproducing strains of $B$. cinerea are used for biotechnological production of ABA (Tan and Li, 1998). Recently, an ABA gene cluster that contains gene bcaba1, bcaba2, bcaba3, and bcaba4 in $B$. cinerea SAS56 was identified (Siewers et al. 2004; Siewers et al. 2006).

The Whole Genome Shotgun (WGS) sequences of $B$. cinerea B05.10 are public available at http://www.ncbi.nlm.nih.gov/sutils/genom_table.cgi?organi $\mathrm{sm}=$ fungi. In this work, the putative ABA gene cluster of $B$. cinerea B05.10 was identified by an in silico genomic sequence analysis. To our surprise an impressive divergence in structure of the two ABA gene clusters was identified. An LTR retrotransposon and a solo LTR were detected within the ABA gene cluster in B. cinerea B05.10, but not in $B$. cinerea SAS56. For it has been shown that retrotransposons may contribute to the expression pattern of many host genes (Kashkush et al. 2003), this divergence in structure of the two ABA gene clusters putatively gives new clues to survey the divergence in the ABA production yields of different $B$. cinerea strains.

\section{MATERIALS AND METHODS}

Nucleotide sequences of bcaba1(AJ609392), bcaba2 (AJ851088), bcaba3 (AM237449) and bcaba4 (AM237450) were obtained from GenBank and used to query the whole genome sequence (WGS) of $B$. cinerea B05.10 by BLASTN search provided by the Broad Institute (Altschul et al. 1997). The annotated features of the genome sequences from the bcaba4 gene to the bcaba3 gene were viewed using Browse Region provided by the Broad Institute. The scheme of the putative ABA gene cluster of $B$. cinerea B05.10 was drawn by the computer software Chem Draw ultra 8.0.

The DNA sequence of B. cinerea B05.10 from the bcaba4 gene to the bcaba3 gene was extracted using Browse Region provided by the Broad Institute. The repetitive elements were analyzed in RepeatMasker. The termini of the LTRs were confirmed by manual inspection. Protein domains were identified using RPSBLAST (MarchlerBauer and Bryant, 2004).

Sequences of Saccharomyces cerevisiae tRNAs used for identification of PBSs were obtained from the Genomic tRNA Database (http://lowelab.ucsc.edu/GtRNAdb/Scere) (Lowe and Eddy, 1997). The polypurine tract (PPT) and the target-site duplications (TSD) of LTR retrotransposon were investigated by manual inspection.

\section{RESULTS AND DISCUSSION}

The putative ABA gene cluster of $B$. cinerea B05.10 is located in the supercontig 41 from 72143 to 91420 bp (in the cont1.1706, NZ_AAID01001706). Sequence identity analysis reveals that each ABA gene of strain B05.10 shows a high degree of similarity to the ABA gene of strain SAS56 (gene bcaba1, bcaba2 and bcaba4 with 99\% identity, respectively; gene bcaba 3 with $98 \%$ identity). It is noteworthy that an astonishing divergence in structures of the two ABA gene clusters was identified. In $B$. cinerea SAS56, gene bcaba3 is located $3.7 \mathrm{~kb}$ upstream of gene bcaba1 (Figure 1a), while in B. cinerea B05.10, gene 
a

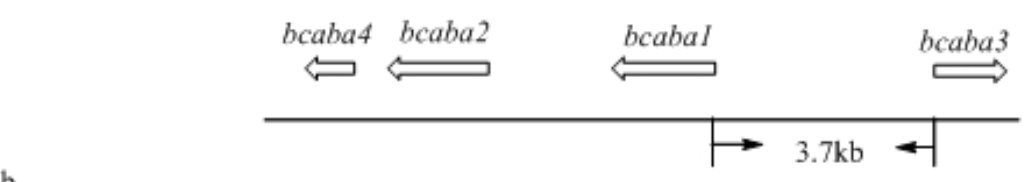

b

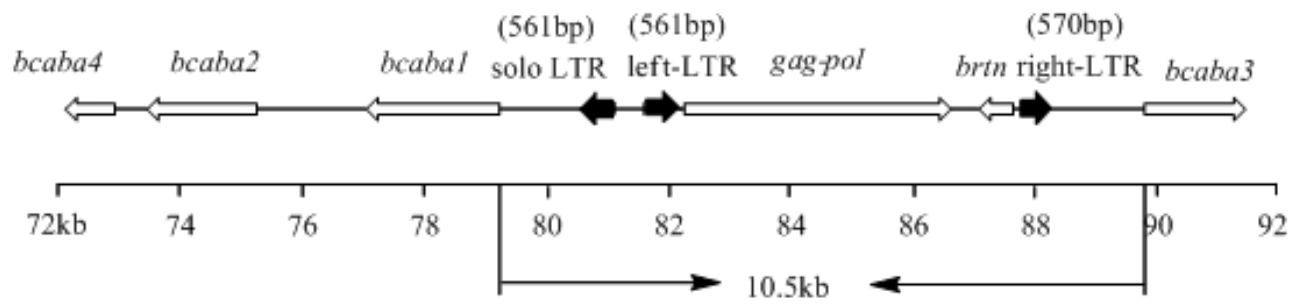

$1 \mathrm{~kb}$

GAG-POL

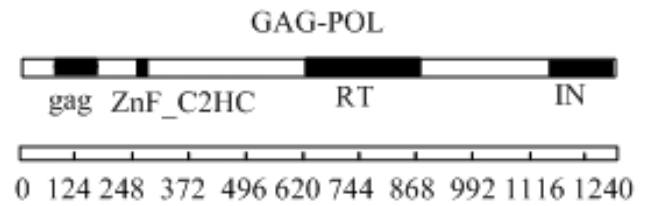

Figure 1. Representations of the structural features of :

(a) The ABA gene cluster in B. cinerea strain SAS56 (Siewers et al. 2006).

(b) The ABA gene cluster in B. cinerea strain B05.10.

(c) Protein GAG-POL of Boty-aba.

DNA depicted with _ $\ldots$, Genes were depicted with the $\vec{l}$, LTRs is indicated with $\overrightarrow{\mathbf{C}}$, and the putative conserved domains is indicated with

bcaba3 is located about $10.5 \mathrm{~kb}$ upstream of gene bcaba1 and two putative genes were detected within this region. A sequence similarity search by BLASTP and BLASTN revealed that the first gene is an LTR retrotransposon gagpol gene, and the second is a novel gene (Figure 1b).

To investigate the structure features of ABA gene cluster of $B$. cinerea B05.10, about $20 \mathrm{~kb}$ of WGS sequences of $B$. cinerea B05.10 from gene bcaba4 to gene bcaba3 was extracted. The extract was analyzed with RepeatMasker. A solo LTR and a LTR retrotransposon that was designated as Boty-aba were identified.

Boty-aba is flanked by 5 bp direct repeats (CATTC) representing target-site duplications (TSD). It contains 6603bp with a pair of LTRs flanking and two internal deduced genes. The LTRs contain 561bp (left) and 570bp (right), respectively. And they share 93.8\% identity. A sequence similarity search by BLASTN demonstrates that both left and right LTR show significant similarity to the LTR of Boty ( $\underline{\text { X81790)}}$, with $80.2 \%$ and $82.0 \%$ identity, respectively. Structure factures analysis reveals that the three LTRs contain the 5'-terminal and 3'-terminal sequences (5'TG...CA3') and perfect short inverted terminal repeats of 7 bp (TGTTACG...CGTAACA) (Diolez et al. 1995). The presumed TATA boxes are found in the three LTRs. Boty-aba and Boty contain the identical primer binding sites (PBSs) for first-strand reverse transcription with 9 nt (5'-TTTGAGCAC-3') immediately downstream of the left LTR. They both use self-priming mechanism to initiate synthesis of reverse transcripts (Lin and Levin, 1997). The polypurine-rich sequence that corresponds to the primer binding site for plus-strand DNA (PPT) synthesis is located immediately upstream of the right LTR, and the sequence of PPT in Boty-aba is 5'AGGCTAAGAAGGGGATAG-3'. The solo LTR is located 448 bp upstream of Boty-aba and flanked by 5 bp direct repeats (CTCAT) representing TSD; it is inverted and is identical with the left-LTR of Boty-aba.

The first internal gene of LTR retrotransposon is a gag-pol gene. This gene has the same transcription direction as gene bcaba 3 and contains 4255 nucleotides with 3 exons coding for 1302 amino acids. Conserved domain search analysis by RPSBLAST demonstrates that this gene encodes a polyprotein with a retrotrans_gag (gag) domain, a C2HC zinc fingers, a reverse transcriptase (RT) domain, and an integrase core (IN) domain (Figure 1c). This polyprotein does not contain protease and RNase $\mathrm{H}$ domains. The analysis of the conserved domains by RPSBLAST is shown in Figure 2.The fact that the domains of Boty-aba within the pol gene are arranged in the order RT and IN reveals that Boty-aba belongs to the Ty3/gypsy group of retrotransposons. The fact that the LTRs of Boty-aba are not identical and there are no protease and RNase $\mathrm{H}$ domains suggests that Boty-aba is an ancient element and it possibly cannot retrotranspose. 


\section{a}

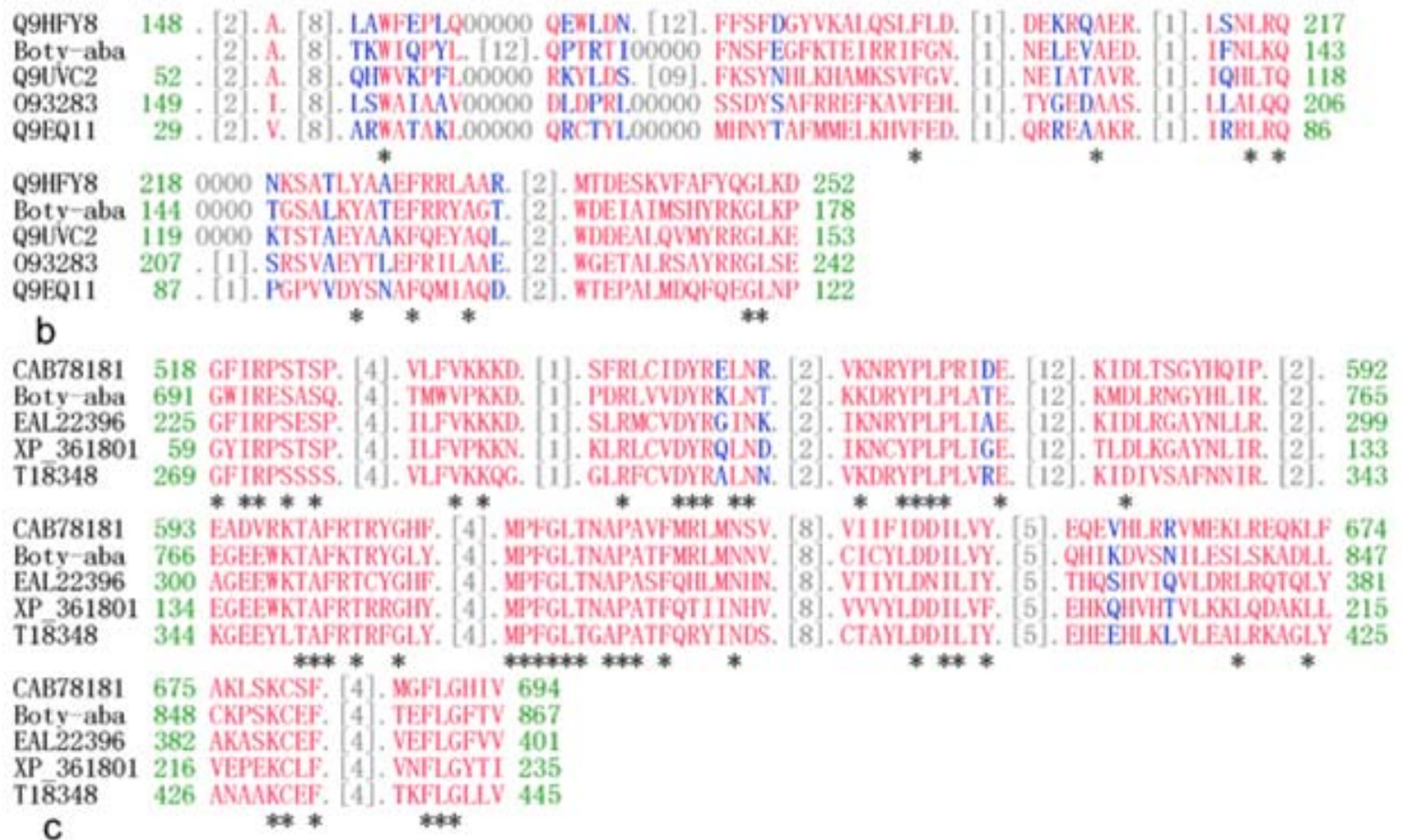

1BL3 C 4. [1]. VDCSPGIWOL.DCTIILEG00000 KVIL.VAVIVASGYTEAEVIPAETCOETAYFLL.KLAO000 GRW. [1]. VKTV 64

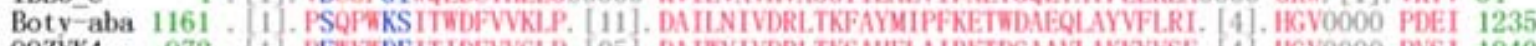

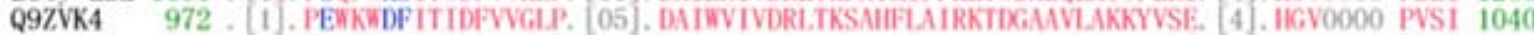
064892591 . (1). PVWKWEKITMDFVTGI.P. (06). DAIWVIVDRL.TKSAHFIPIIITTITGERLAQVYI.DE. 4 4. HGV0000 PTSI 660

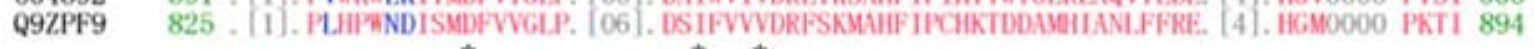

1B.3 C 65 IITDNG. [1]. NFTSTTVKMCWWAGIKQEFGIPYNPQSQGVIESMNKEI.KKIIO000 6Q. [36]. 151 Boty aba 1236 ISDRD. (1). I.FTSKFWTTLIALMGIKRKISTSFIIPQTDGQTERTNQTMEAYI. [2]. YR. (14). 1302

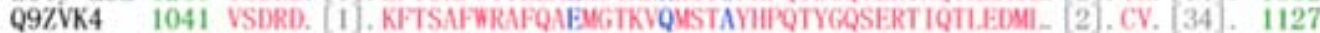
064892661 VSDRD, 1 1 . RFVSHFWRSLODAL.GTRL.DFSTAFHPQSDGQSERTIOTLFDML. 22. CV. 34.747 Q9ZPF9 895 VSDRD, [1]. KFLSYFWKTL.WSKL.GTKL.LFSTTCHPQTDCQTEVVNRTLSTLL. [2]. L.I. [34]. 981 * * * * * * *

Figure 2. Analysis of the:

(a) gag domain.

(b) RT domain.

(c) IN domain of the Boty-aba GAG-POL protein.

Absolutely conserved amino acids are indicated with an asterisk $\left(^{*}\right)$, identical residues are coloured red, similar residues are coloured blue, masked out regions are coloured gray. Q9HFY8 is the gag protein of an LTR-retrotransposon (Cgret) from Glomerella cingulata; Q9UVC2 is the gag polyprotein of an LTR-retrotransposon (CfT-I) in Cladosporium fulvum; O93283 is the gag polyprotein of the LTR retrotransposon from Takifugu rubripes; Q9EQ11 is the gag domain of Myelin expression factor-3-like protein from Mus musculus; CAB78181 is the putative reverse-transcriptase-like protein from Arabidopsis thaliana; EAL22396 is the RT domain of the hypothetical protein CNBB2750 in Cryptococcus neoformans var. neoformans B-3501A; XP_361801 is the RT domain of the hypothetical protein MG04275.4 in Magnaporthe grisea 70-15; T18348 the RT domain of the gypsy retrotransposon in Magnaporthe grisea; 1BL3 C is the catalytic domain of HIV-1 integrase; Q9ZVK4 is the integrase core domain of the putative retroelement pol polyprotein from Arabidopsis thaliana; O64892 is the integrase core domain of the polyprotein from Ananas comosus; Q9ZPF9 is the IN domain of the F5K24.1 protein (putative polyprotein) from Arabidopsis thaliana.

Retrotransposons are a widespread and important class of eukaryotic mobile genetic elements that have a central role in the structure, evolution, and function of eukaryotic genomes (Bennetzen, 2000; Kidwell and Lisch, 2001). Recent reports have shown that retrotransposons contribute to the formation of genome structure and to the expression pattern of many host genes (Kashkush et al. 2003). For example, when Wis 2-1A retrotransposons are activated in wheat, the expression of several adjacent genes is activated or silenced by producing sense or antisense transcripts of those genes (Kashkush et al. 2003). Matsubara concluded that at least 3 transposable elements in $\mathrm{Hf} 1$ gene that plays a key role in the expression of floral colour in petunias govern anthocyanin biosynthesis of commercial petunias (Matsubara et al. 2005). It is not known whether Boty-aba has an affect on the expression of ABA genes. This divergence in structure of the ABA gene clusters gives new clues to survey the divergence in the ABA production yields of different $B$. cinerea strains. 
The second internal gene of LTR retrotransposon is 597 bp long and encodes 199 amino acids. It is transcribed divergently. No putative conserved domain has been detected using RPSBLAST. On the basis of these results, we believe that this is a novel gene, which we designate brtn (B. cinerea retrotransposon novel) and the deduced protein is named BTRN. The structural features of Boty$a b a$ are depicted in Figure 1b. We also identified an EST (W0AA017ZF01C1) shows high sequence similarity to gene brtn (100\% identities). We presume that the internal promoter of the flanking LTRs drive the transcription of gene $b r t n$.

Retrotransposons are a widespread and important class of eukaryotic mobile genetic elements that have a central role in the structure, evolution, and function of eukaryotic genomes (Bennetzen, 2000 ; Kidwell and Lisch, 2001). Recent reports have shown that retrotransposons contribute to the formation of genome structure and to the expression pattern of many host genes (Kashkush et al. 2003). For example, when Wis 2-1A retrotransposons are activated in wheat, the expression of several adjacent genes is activated or silenced by producing sense or antisense transcripts of those genes (Kashkush et al. 2003). Matsubara concluded that at least 3 transposable elements in Hf1 gene that plays a key role in the expression of floral colour in petunias govern anthocyanin biosynthesis of commercial petunias (Matsubara et al. 2005). It is not known whether Boty-aba has an affect on the expression of ABA genes. This divergence in structure of the ABA gene clusters gives new clues to survey the divergence in the $\mathrm{ABA}$ production yields of different $B$. cinerea strains.

\section{REFERENCES}

ALTSCHUL, S.F.; MADDEN, T.L.; SCHÄFFER, A.A.; ZHANG, J.H.; ZHANG, Z.; MILLER, W. and LIPMAN, D.J. Gapped BLAST and PSIBLAST: a new generation of protein search programs. Nucleic Acids Research, September 1997, vol. 25, no. 17, p. 3389-3402.

BENNETZEN, J.L. Transposable element contributions to plant gene and genome evolution. Plant Molecular Biology, January 2000, vol. 42, no. 1, p. 251-269.

DIOLEZ, A.; MARCHES, F.; FORTINI, D. and BRYGOO, Y. Boty, a long-terminal-repeat retroelement in the phytopathogenic fungus Botrytis cinerea. Applied and Environmental Microbiology, January 1995, vol. 61, no. 1, p. 103-108.

KASHKUSH, K.; FELDMAN, M. and LEVY, A.A. Transcriptional activation of retrotransposons alters the expression of adjacent genes in wheat. Nature Genetics, January 2003, vol. 33, no. 1, p. 102-106.

KIDWELL, M.G. and LISCH, D.R. Perspective: transposable elements, parasitic DNA, and genome evolution. Evolution, January 2001, vol. 55, no. 5, p. 1-24.
LIANG, Y.; ZHENG, H.; WU, L. and WANG, M. Effect of carotenoids on abscisic acid production of Botrytis cinerea. Pharmaceutical Biotechnology, April 2004, vol. 11, no. 2, p. 96-98.

LIN, J.H. and LEVIN H.L. Self-primed reverse transcription is a mechanism shared by several LTRcontaining retrotransposons. RNA, September 1997, vol. 3, no. 9, p. 952-953.

LOWE, T.M. and EDDY, S.R. tRNAscan-SE: A program for improved detection of transfer RNA genes in genomic sequence. Nucleic Acids Research, March 1997, vol. 25, no.1, p. 955-964.

MARCHLER-BAUER, A. and. BRYANT, S.H. CDSearch: protein domain annotations on the fly. Nucleic Acids Research, July 2004, vol. 32, p. 327-331.

MATSUBARA, Kiyoshi; KODAMA, Hiroaki; KOKUBUN, Hisashi; WATANABE, Hitoshi and ANDO, Toshio. Two novel transposable elements in a cytochrome P450 gene govern anthocyanin biosynthesis of commercial petunias. Gene, September 2005, vol. 358, p. 121-126.

SIEWERS, V.; SMEDSGAARD, J. and TUDZYNSKI, P. The P450 monooxygenase bcaba1 is essential for abscisic acid biosynthesis in Botrytis cinerea. Applied and Environmental Microbiology, July 2004, vol. 70, no. 7, p. 3868-3876.

SIEWERS, V.; KOKKELINK, L.; SMEDSGAARD, J. and TUDZYNSKI, P. Identification of an abscisic acid gene cluster in the grey mold Botrytis cinerea. Applied and Environmental Microbiology, July 2006, vol. 72, no. 7, p. 4619-4626.

TAN, H. and LI, Z.D. Method for producing natural abscisic acid by fungus fermentation. Chinese Patent, 1998, CN1182798.

TUDZYNSKI, B. and SHARON, A. Biosynthesis biological role and application of fungal hormones. In: OSIEWACZ, H.D. eds. The mycota $X$ : industrial applications. Springer-Verlag, Berlin, Germany, 2002, p. 183-211. ISBN 3540415831.

WU, J. and SHI, Z. Isolation of abscisic acid producing strains of phytopathogens. Phytochemistry, September 1998, vol. 49, no. 1, p. 89-90.

WU, J. and ZHENG, H. Microbial production of abscisic acid by phytopathogenic fungus Botrytis cinerea A 23. Journal of China Pharmaceutical University, October 1997, vol. 28, no. 5, p. 313-316. 